\title{
Efeitos do Octil-metoxicinamato na Histopatologia do Testículo, Rim e Fígado de Ratos Wistar
}

\author{
Effects of Octyl methoxycinnamate on the Histopathology of Testicle, \\ Kidney and Liver in Wistar Rats
}

\author{
Vitor Kunes Lopes ${ }^{1}$ \\ Ana Rosa Crisci
}

\section{RESUMO}

Objetivo: Investigar os efeitos adversos que o OMC pode causar nas funções reprodutiva, hepática e renal, por meio da análise comparativa da morfologia dos testículos, rins e fígados, qualidade do esperma e alterações na massa corporal de ratos Wistar. Metodologia: Os animais, com 2 meses de idade, foram divididos em dois grupos, os quais o primeiro grupo, controle, ingeriu apenas óleo de milho, a partir de uma dose inicial de $0,6 \mathrm{ml}$ e o segundo grupo, tratado, ingeriu apenas o OMC, com a mesma dose inicial. As doses foram ajustadas diariamente com base no peso atual do animal, em uma proporção de $5 \mathrm{ml} / \mathrm{kg}$. Após 21 dias de exposição às substâncias, os animais foram sacrificados e foi realizada a laparotomia para coleta de testículos, rins e fígado para análise histopatológica, além de amostras de esperma para elaboração do espermograma. Resultados: A análise histopatológica evidenciou áreas hemorrágicas no parênquima do córtex renal, alterações celulares nos cordões de hepatócitos, perda dos tubos seminíferos e alterações nas células de linhagem espermatogênica. A análise do espermograma comprovou que a substância causou um aumento na taxa de espermatozoides anormais, com aumento no número de anomalias na cabeça e cauda. Conclusão: Foi constatado que o filtro solar analisado, a partir das alterações morfológicas nos órgãos e na qualidade do sêmen, de fato afetou as funções reprodutivas, hepáticas e renais dos animais analisados.

\section{DESCRITORES}

Filtro solar. Octil-Metoxicinamato. Histopatologia. Espermograma.

\begin{abstract}
Objective: To investigate the adverse effects that $\mathrm{OMC}$ can cause in reproductive, liver and renal functions, through the comparative analysis of the morphology of the testicles, kidneys and livers, sperm quality and changes in the body mass of Wistar rats. Methodology: The animals, with 2 months of age, were divided in two groups, in which the control ingested only corn oil, from an initial dose of $0.6 \mathrm{ml}$, and the treatment group ingested only the OMC, with the same initial dose. The doses were adjusted daily based on the current weight of the animal, in a proportion of $5 \mathrm{ml} / \mathrm{kg}$. After 21 days of exposure, the animals were sacrificed and the laparotomy was made from the testicles, kidneys, and livers for histopathological analysis, besides sperm samples for elaboration of the spermogram. Results: The histopathological analysis evidenced hemorrhagic areas in the renal cortex parenchyma, cellular alterations in the hepatocyte strands, loss of seminiferous tubes and alterations in the cells of spermatogenic lineage. The spermogram analysis proved that the substance caused an increase in the abnormal sperm rate, with an increase in the rate of anomalies in the head and tail. Conclusion: It was found that the analyzed solar filter, from morphological alterations in the organs and sperm quality, in fact affected the reproductive, liver, and renal functions in the analyzed animals.
\end{abstract}

\section{DESCRIPTORS}

Solar filter. Octyl-Metoxycinnamate. Histopathology. Spermogram.

${ }^{1}$ Graduando em Ciências Biológicas pelo Centro Universitário Barão de Mauá, Ribeirão Preto, São Paulo, Brasil.

${ }^{2}$ Mestre em Bioengenharia pela Universidade de São Paulo - USP, São Paulo, Brasil. 
$\mathrm{O}$ filtros solares são compostos lipofílicos que atua como bioacumuladores nos tecidos dos animais aquáticos ${ }^{1}$. Seus efeitos são similares aos demais compostos xenobióticos, causando alterações comportamentais, reprodutivas e hormonais nos animais expostos ${ }^{2}$.

O crescente uso de filtros solares pela população em regiões costeiras e ribeirinhas contribui para a contaminação da água, peixes e até de alimentos por meio da irrigação'. Além disso, trabalhos apontam a presença de substâncias derivadas de filtros solares em águas de torneira e de esgoto tratado ${ }^{3}$.

O octil-metoxicinamato (OMC) é um dos filtros solares mais utilizados em cosméticos atualmente na proteção contra os efeitos nocivos dos raios ultravioleta. Os estudos sobre essa substância começaram quando ela foi a protagonista do branqueamento dos corais que ocorreu na Oceania ${ }^{4}$. Porém, já existiam estudos que relatavam seus efeitos nas funções hormonais de mamíferos ${ }^{5}$.

Existem relatos da presença do OMC em fluidos biológicos como o sangue, a urina e o leite materno, além de ser apontado como possível causador de vasorelaxamento nas artérias umbilicais e potencial desregulador da tireoide ${ }^{6}$.

O comprometimento da função tireoidiana é causado pela exposição à desreguladores endócrinos, levando à distúrbios neurológicos e problemas de desenvolvimento fetal, além do hipotireoidismo ${ }^{7}$. O hipotireoidismo é um distúrbio que prejudica a produção do hormônio tiroxina pela glândula tireoide, desacelerando o metabolismo, gerando fadiga, sonolência, depressão e raciocínio lento ${ }^{8}$.
A desregulação endócrina é um mecanismo que possui efeitos relacionados ao funcionamento do sistema endócrino, tendo potencial de danificar ou alterar a função de um órgão endócrino, como os rins, fígados e glândulas sexuais, interagir com um receptor de hormônios ou alterar o metabolismo de um hormônio produzido? .

Diversos potenciais desreguladores endócrinos têm sido constantemente lançados no ambiente durante os últimos anos, sendo 0 ambiente aquático o mais afetado ${ }^{10}$. Os seres humanos podem ser expostos à essas substâncias de diferentes formas, principalmente de maneira indireta, pela ingestão de água ou alimentos contaminados ${ }^{9}$.

Os efeitos de desreguladores endócrinos, como é o caso do OMC, nas funções reprodutivas e hepáticas já são em parte conhecidos ${ }^{11}$. A exposição ao OMC em machos causa relativa redução de peso dos testículos e uma diminuição da produção do hormônio testosterona ${ }^{12}$. Além de efeitos nos testículos, a diminuição na produção do hormônio tiroxina ocasiona alterações nas atividades enzimáticas do fígado ${ }^{11}$.

Pela pesquisa bibliográfica preliminar, foi notado que embora alguns dos efeitos do OMC nos testículos e fígado sejam relatados, existe uma escassez de trabalhos que analisem as alterações morfológicas ocasionadas nos tecidos desses órgãos e não existem relatos de efeitos nos rins. A literatura também carece de análises da qualidade de esperma dos animais analisados, comprovando a necessidade de ampliar as pesquisas nessas áreas.

Com base nisso, os objetivos do estudo em questão foram analisar as alterações 
morfológicas que o OMC pode causar nos tecidos dos rins, fígados e testículos em ratos Wistar, além de descrever alterações na massa corporal e na qualidade do esperma, com o intuito de evidenciar possíveis alterações nas funções reprodutiva, hepática e renal dos animais.

\section{METODOLOGIA}

Foram utilizados 20 ratos Wistar machos, recém desmamados, com 60 dias e peso médio inicial de $250 \mathrm{~g}$. Durante o período experimental os animais foram mantidos no biotério em condições controladas de temperatura $\left(20\right.$ e $25^{\circ} \mathrm{C}$ ) e umidade relativa (55\%), em regime de luz de $12 \mathrm{~h} / 12 \mathrm{~h}$, sendo acesas às 08:00 h e apagadas às 20:00 h. Os animais receberam ração balanceada e água ad-libitum.

Depois de identificados, os animais foram divididos aleatoriamente em dois grupos (controle e tratado) com 10 animais cada, onde conviveram dois animais por caixa.

O grupo controle recebeu uma dose inicial de $0,6 \mathrm{ml}$ de óleo de milho, onde a dose foi ajustada diariamente de acordo com a variação de peso do animal, na qual a dose final foi de $0,71 \mathrm{ml}$. O grupo tratado recebeu uma dose inicial de $0,6 \mathrm{ml}$ do OMC, também ajustada diariamente, na qual a dose final foi de $0,63 \mathrm{ml}$

As doses foram realizadas uma vez por dia, durante 21 dias, no período da tarde, por via oral com sonda gástrica apropriada (gavagem $)^{13}$. Os animais foram pesados ao final de cada dia para que a dosagem fosse ajustada de acordo com o seu peso atual, em uma proporção de $5 \mathrm{ml} / \mathrm{kg}^{14}$.
Após o período de exposição às substâncias, os animais foram anestesiados com injeção de Tiopental sódico na dose de $120 \mathrm{mg} / \mathrm{kg}$ IP. Após o sacrifício foi realizada a laparotomia para a coleta de testículos, rins, e fígados, onde apenas os testículos foram pesados para posterior comparação, tendo em vista os relatos de alterações na massa desses órgãos pela desregulação hormonal ${ }^{12}$.

Foram preparadas lâminas individuais para cada órgão dos animais do grupo controle e tratado, com cortes de $8 \mu \mathrm{m}$ de espessura, fixados em formalina $10 \%$, incluídos em parafina histológica e corados com hematoxilina-eosina ${ }^{15}$.

Além dos órgãos, foram coletadas amostras de esperma para elaboração do espermograma. A amostra de esperma foi separada e diluída em $1 \mathrm{ml}$ de solução fisiológica, sendo observada no microscópio óptico para classificação do nível de vigor dos espermatozoides de cada animal. Para analisar a morfologia dos espermatozoides foi utilizado o método Eosina-Nigrosina, onde foram adicionadas 1 gota de esperma homogeneizado, coletadas 30 minutos antes, 2 gotas de Eosina 3\% e 4 gotas de solução de Nigrosina $8 \%$ para a preparação das lâminas ${ }^{16}$. O esfregaço foi observado em microscópio óptico com objetiva de imersão, contando 100 espermatozoides por lâmina.

\section{ANÁLISE DOS DADOS}

Após a coleta dos órgãos foram realizadas suas devidas análises histopatológicas, onde foram comparadas as diferenças existentes nos tecidos dos grupos controle e tratado para identificar possíveis alterações morfológicas ocasionadas pela 
ingestão do OMC. Além da análise comparativa dos órgãos, a variação do peso dos animais e de seus respectivos testículos foi equiparada.

Por meio do Test-t Student para amostras independentes, foram comparados os pesos dos animais e os testículos dos dois grupos e por meio do teste para amostras pareadas, os pesos de animais do mesmo grupo $^{12}$. Foram comparadas também as amostras de esperma de ambos os grupos, por meio do Test-t Student para amostras independentes, onde foram aferidas diferenças no vigor e na taxa de normalidade dos espermatozoides.

\section{RESULTADOS}

$\mathrm{Na}$ análise histopatológica do rim dos animais do grupo controle, observou-se que o parênquima do córtex renal apresentava aspectos normais, com glomérulos renais e estruturas tubulares apresentando núcleos com cromatina frouxa (Fig. 1A). No grupo que ingeriu $\mathrm{OMC}$, o parênquima do córtex renal apresentou áreas hemorrágicas intra e extraglomerulares (Fig. 1B, seta preta).

Observou-se, na análise histopatológica do fígado do animal do grupo controle, que o parênquima hepático estava normal, com aspectos lobulares preservados e cordões de hepatócitos com características também normais (Fig. 1C). No entanto, no grupo experimental que ingeriu o OMC, verificaram-se áreas pouco preservadas com evidente destrabeculação hepatocelular, discreto infiltrado inflamatório com congestão vascular (Fig. 1D, seta preta), hepatócitos com degeneração hidrópica (Fig. 1D, seta

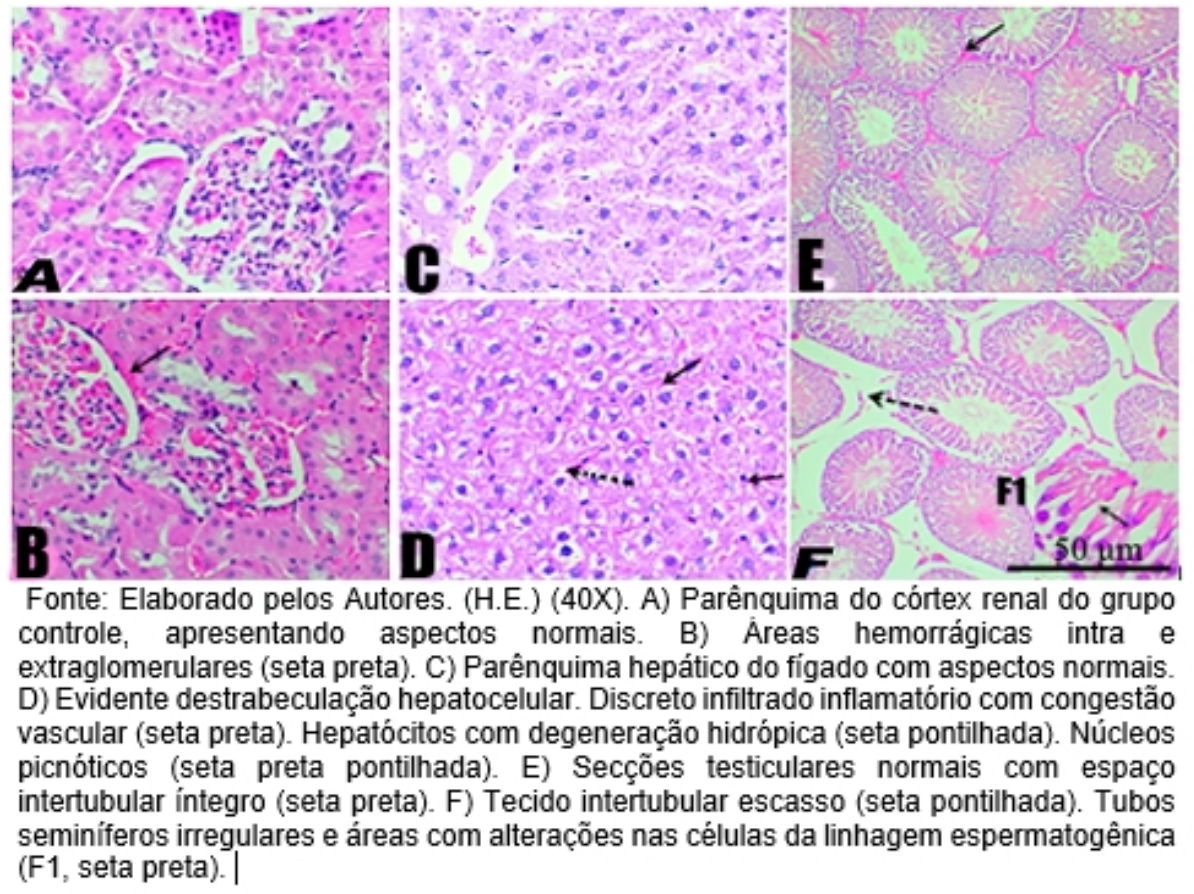


Tabela 1. Comparação da taxa de mobilidade dos espermatozoides dos animais do Grupo Controle e Tratado

\begin{tabular}{c|c|c}
\cline { 2 - 3 } & \multicolumn{2}{|c}{ Taxa de Mobilidade } \\
\hline Animal & Controle & Tratado \\
\hline I & 01 & - \\
II & - & 03 \\
III & 04 & 03 \\
IV & 05 & 04 \\
V & 05 & 02 \\
VI & 03 & 02 \\
VII & 03 & 02 \\
VIII & 05 & 05 \\
IX & 04 & 02 \\
X & 05 & 01 \\
\hline
\end{tabular}

Fonte: Elaborado pelos Autores.

pontilhada) e núcleos picnóticos (Fig. 1D, seta preta curta).

Nas secções testiculares dos ratos do grupo controle (Fig. 1E), os tubos seminíferos apresentaram-se bem organizados. O epitélio seminífero mostrou-se íntegro, com células espermatogênicas em todas as etapas de desenvolvimento e o espaço intertubular apresentou-se normal, contendo vasos sanguíneos (Fig. 1E, seta preta). Nas secções testiculares dos ratos do grupo tratado com OMC (Fig. 1F), os tubos seminíferos apresentaram-se irregulares e com áreas com alterações nas células da linhagem espermatogênica (Fig. 1F, F1, seta preta), e o tecido intertubular apresentou-se bem escasso (Fig. 1F, seta pontilhada).

\section{Espermograma}

Com base na análise comparativa

Figura 2. Alterações morfológicas no esfregaço de espermatozoides
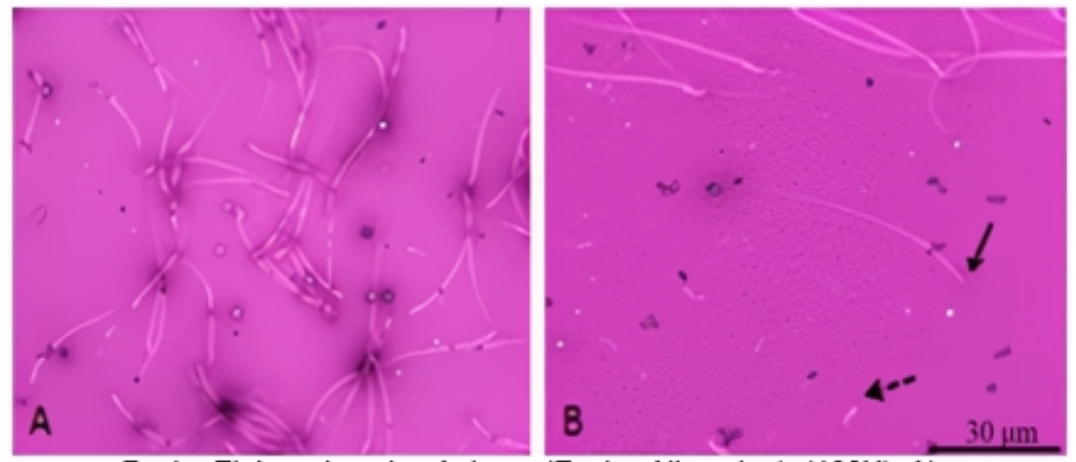

Fonte: Elaborado pelos Autores.(Eosina-Nigrosina). (100X). A)

Espermatozoides do grupo controle apresentaram aspectos normais. B)

Espermatozoides anormais de animais que ingeriram o OMC.

Sem cauda (seta pontilhada) e acéfalo (seta preta). 
do esperma dos dois grupos por meio do espermograma, se observou que a taxa de mobilidade (vigor) média dos espermatozoides dos grupos controle e tratado não foram significativamente diferentes ( $p=0,1449)$.

Ao verificar a morfologia dos espermatozoides, notou-se que as taxas médias de normalidade dos espermatozoides dos grupos controle e tratado foram de fato significativamente diferentes ( $p=0,0428)$, onde foi verificado um aumento na taxa de espermatozoides acéfalos (Fig. 2B, seta preta) e sem cauda (Fig. 2B, seta pontilhada) no grupo que recebeu o OMC. As taxas que indicavam espermatozoides sem gancho, com cauda enrolada, cauda com angulação

Tabela 2. Taxas de normalidade e anomalias dos espermatozoides dos animais do Grupo Controle

\begin{tabular}{|c|c|c|c|c|c|c|c|c|c|}
\hline \multirow[t]{2}{*}{ C } & \multirow[t]{2}{*}{$\mathrm{N}$} & \multirow{2}{*}{$\begin{array}{c}\begin{array}{c}\text { Anomalias } \\
\text { De } \\
\text { Cabeça }\end{array} \\
\text { ACF }\end{array}$} & \multicolumn{5}{|c|}{$\begin{array}{c}\text { Anomalias } \\
\text { De } \\
\text { Cauda }\end{array}$} & \multirow{2}{*}{$\begin{array}{c}\text { Anomalias } \\
\text { De } \\
\text { Gancho }\end{array}$} & \multirow{2}{*}{$\begin{array}{c}\begin{array}{c}\text { Anomalias } \\
\text { Múltiplas }\end{array} \\
\text { SG + SC }\end{array}$} \\
\hline & & & SC & $\mathrm{CE}$ & CAA & CAT & SA & & \\
\hline \multicolumn{10}{|c|}{$\%$} \\
\hline $\mathrm{C} 1$ & 90 & 02 & 04 & 01 & -- & - & 02 & - & 01 \\
\hline C2 & 83 & 04 & 07 & 01 & -- & 01 & 03 & -- & 01 \\
\hline C3 & 81 & 04 & 08 & 02 & 01 & -- & 02 & -- & 02 \\
\hline $\mathrm{C} 4$ & 79 & 05 & 11 & 02 & 01 & -- & 01 & -- & 01 \\
\hline C5 & 90 & 04 & 05 & - & - & -- & 01 & -- & -- \\
\hline C6 & 89 & 08 & 02 & - & - & - & 01 & - & - \\
\hline C7 & 87 & 03 & 06 & 01 & -- & - & 01 & 01 & 01 \\
\hline C8 & 90 & 04 & 03 & 01 & -- & 02 & - & -. & -- \\
\hline C9 & 75 & 09 & 10 & 02 & 02 & -- & 01 & 01 & -- \\
\hline C10 & 93 & 03 & 02 & - & - & - & 02 & - & - \\
\hline
\end{tabular}

Tabela 3. Taxas de normalidade e anomalias dos espermatozoides dos animais do Grupo Tratado

\begin{tabular}{|c|c|c|c|c|c|c|c|c|c|}
\hline \multirow[t]{2}{*}{$\mathrm{T}$} & \multirow[t]{2}{*}{$\mathrm{N}$} & \multirow{2}{*}{$\begin{array}{c}\text { Anomalias } \\
\text { De } \\
\text { Cabeça }\end{array}$} & \multicolumn{5}{|c|}{$\begin{array}{c}\text { Anomalias } \\
\text { De } \\
\text { Cauda }\end{array}$} & \multirow{2}{*}{ 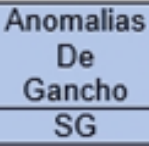 } & \multirow{2}{*}{$\begin{array}{c}\text { Anomalias } \\
\text { Múltiplas }\end{array}$} \\
\hline & & & SC & $\mathrm{CE}$ & CAA & CAT & SA & & \\
\hline \multicolumn{10}{|c|}{$\%$} \\
\hline T1 & 35 & 21 & 41 & -- & - & - & 01 & - & 02 \\
\hline T2 & 88 & 02 & 06 & -- & 02 & - & 02 & - & -- \\
\hline T3 & 76 & 09 & 12 & 02 & - & -. & 01 & - & -- \\
\hline $\mathrm{T} 4$ & 74 & 04 & 21 & - & - & - & 01 & - & - \\
\hline T5 & 64 & 05 & 20 & 05 & 04 & 01 & 01 & - & - \\
\hline T6 & 65 & 12 & 23 & - & - & - & - & - & -- \\
\hline T7 & 90 & 07 & 02 & -- & - & -- & 01 & - & -- \\
\hline T8 & 90 & 02 & 03 & 04 & - & 01 & -- & -. & -- \\
\hline T9 & 78 & 08 & 11 & - & - & - & 02 & -- & 01 \\
\hline T10 & 71 & 11 & 14 & 01 & 02 & - & 01 & - & - \\
\hline
\end{tabular}


acentuada, cauda com angulação atenuada, cauda sem angulação ou sem gancho e sem cauda permaneceram normais.

Variação de peso dos animais

Ao analisar a variação de peso dos animais do grupo controle, concluiu-se que o ganho médio de peso dos animais desse grupo foi de $24,3 \mathrm{~g}$, onde esse ganho médio não foi estatisticamente significativo ( $p=$ 0,0981 ). Ao observar a variação de peso dos animais do grupo tratado, concluiu-se que o ganho médio de peso dos animais desse grupo foi de $27,9 \mathrm{~g}$, onde esse ganho médio de peso foi estatisticamente significativo $(p=$ 0,0131).

Ao comparar os pesos finais do grupo controle e do grupo tratado, foi realizado que os pesos finais médios dos animais dos dois grupos

Tabela 4. Comparação entre os pesos iniciais e finais dos animais do Grupo Controle e Tratado

\begin{tabular}{c|c|c|c|c}
\cline { 2 - 5 } & \multicolumn{2}{c|}{ Grupo Controle } & \multicolumn{2}{c}{ Grupo Tratado } \\
\hline Animal & Peso Inicial (g) & Peso Final (g) & Peso Inicial (g) & Peso Final (g) \\
\hline I & 271 & 317 & 248 & 257 \\
II & 255 & 294 & 272 & 303 \\
III & 270 & 220 & 258 & 273 \\
IV & 263 & 308 & 281 & 296 \\
V & 276 & 327 & 266 & 284 \\
VI & 271 & 317 & 264 & 268 \\
VII & 258 & 284 & 247 & 273 \\
VIII & 262 & 295 & 288 & 336 \\
IX & 272 & 217 & 260 & 261 \\
X & 276 & 338 & 261 & 273 \\
\hline
\end{tabular}

Tabela 5. Comparação do peso dos testículos direito e esquerdo entre os animais do Grupo Controle e Tratado

\begin{tabular}{c|c|c|c|c}
\cline { 2 - 5 } & \multicolumn{2}{|c|}{ Grupo Controle } & \multicolumn{2}{c}{ Grupo Tratado } \\
\hline \multirow{2}{*}{ Animal } & $\begin{array}{c}\text { Testículo } \\
\text { Esquerdo (q) }\end{array}$ & $\begin{array}{c}\text { Testículo Direito } \\
(\mathrm{g})\end{array}$ & $\begin{array}{c}\text { Testiculo } \\
\text { Esquerdo (q) }\end{array}$ & $\begin{array}{c}\text { Testículo Direito } \\
(\mathrm{g})\end{array}$ \\
\hline I & 44,47 & 44,43 & 44,61 & 44,47 \\
II & 44,37 & 44,49 & 44,56 & 44,57 \\
III & 44,64 & 44,34 & 44,58 & 44,55 \\
IV & 31,83 & 44,64 & 44,52 & 44,42 \\
V & 44,71 & 44,63 & 44,25 & 44,27 \\
VI & 44,50 & 37,00 & 44,73 & 44,62 \\
VII & 44,68 & 44,37 & 44,48 & 44,57 \\
VIII & 44,65 & 44,52 & 44,50 & 44,53 \\
IX & 44,62 & 44,43 & 44,46 & 44,43 \\
X & 44,59 & 44,45 & 44,51 \\
\hline
\end{tabular}


não foram significativamente diferentes $(p=$ 0,9673).

Variação de peso dos testículos

Ao se comparar os pesos dos testículos direito e esquerdo do grupo controle e do grupo tratado, concluiu-se que os pesos médios dos testículos dos animais dos dois grupos não foram significativamente diferentes $(p=$ 0,3343).

\section{DISCUSSÃO}

Atualmente, existe uma grande utilização de filtros solares possuindo diversos componentes desconhecidos pela opinião popular e, alguns deles, podem ser nocivos tanto à saúde humana quanto para diversos outros organismos ${ }^{14}$. Sendo assim, é de suma importância a investigação sobre os efeitos que essas substâncias podem causar nesses organismos devido à sua grande presença no cotidiano da população, para desvendar os fatores causados pela sua toxicidade e estabelecer medidas de intervenção, no âmbito da saúde pública, quanto ao uso desses compostos.

Os resultados obtidos por este trabalho comprovaram que ocorreram mudanças na estrutura dos órgãos das funções reprodutoras e hepáticas, além de uma queda na qualidade do sêmen, já evidenciadas por Axelstad et al. ${ }^{17}$, sobre os efeitos da exposição ao OMC nos períodos pré e pós-natal, onde alterações nos tecidos dos órgãos foram comprovadas e a taxa de anomalias em espermatozoides aumentou.

As alterações nos rins ocasionadas pelo OMC apresentadas nesse trabalho foram inéditas na literatura, demonstrando a necessidade do estudo das possíveis alterações nos demais órgãos dos organismos afetados, não se limitando apenas às funções hepáticas e reprodutoras.

$\mathrm{Na}$ literatura, os estudos sobre os efeitos da ação dos filtros solares em organismos vivos se distanciam em relação à idade dos ratos e as doses administradas. Os filhotes em fase de amamentação representam o grupo potencialmente mais vulnerável aos mecanismos da toxicidade da substância e não existe um consenso quanto à dose mínima necessária para o início de algum fenômeno tóxico no animal. Neste estudo a dose administrada foi de $5 \mathrm{ml} / \mathrm{kg}$, sendo que doses maiores causaram a mortalidade dos animais em outros estudos ${ }^{14}$.

Os estudos centrados no OMC são em sua maioria focados na ação das substâncias sobre o nível de tiroxina total, onde em grande parte dos casos ocorreu uma queda nesse nível ocasionando casos de hipotireoidismo, evidenciado por uma grande diminuição no ganho de peso e problemas neurológicos posteriores, quando foram utilizados ratos no período lactacional ${ }^{10}$.

Os animais utilizados no presente estudo se encontravam na fase pós-lactacional e foram expostos à substância por um curto período de tempo, gerando menores efeitos sobre as funções hormonais, onde o peso dos animais em geral não sofreu alteração, como evidenciado por Garcia ${ }^{14}$. Além disso, o peso dos testículos dos animais do grupo tratado também não sofreu uma variação significativa, confrontando os dados obtidos por Axelstad, et al. ${ }^{12}$ 
A literatura se expande em diversas áreas, que vão desde alterações comportamentais ocasionadas pelo OMC até os seus efeitos nas artérias umbilicais humanas ${ }^{18}$, evidenciando uma necessidade de ampliar os estudos a respeito dessa substância para chegar a conclusões mais concordantes e abrangentes.

Para novas perspectivas, a aplicação do OMC na pele dos animais, visando analisar os níveis de penetração da substância no sangue, bem como a aplicação de doses menores por via gástrica irão auxiliar no entendimento dos níveis mínimos de exposição em que o OMC é capaz de causar alterações no organismo afetado.

O nosso estudo foi o primeiro a aferir a taxa de mobilidade dos espermatozoides após a exposição. Indicamos a realização do teste de vigor em amostras de esperma coletadas de animais expostos à substância por um período maior, preferencialmente, no período lactacional, para buscar diferenças significativas.

A análise de órgãos não relacionados às funções previamente apresentadas ajudará a compreender os efeitos do OMC nos diferentes sistemas. Um foco principal nas alterações morfofuncionais do cérebro, visto que a literatura relatada alterações comportamentais nos animais expostos.

\section{CONCLUSÃO}

Concluiu-se que a exposição ao OMC durante o período pós-lactacional embora não tenha causado mudanças no ganho de peso, de fato causou alterações morfológicas nos tecidos dos rins, fígados e testículos.

Além de mudanças nas estruturas dos órgãos, foi evidenciado no espermograma que a substância causou um aumento na taxa de espermatozoides anormais e um aumento no número de anomalias na cabeça e na cauda.

O surgimento de alterações morfológicas nos tecidos dos órgãos e o aumento na taxa de espermatozoides anormais comprovam que de fato a substância foi capaz de afetar as funções reprodutivas, hepáticas e renais dos animais expostos.

As alterações causadas pelo OMC sugerem uma necessidade de maiores estudos sobre seus efeitos tendo em vista que essa e outras substâncias estão presentes no cotidiano de grande parte da população, servindo de alerta as pessoas para que não se torne um problema de saúde pública pelo uso descuidado de produtos que contenham grandes doses de OMC.

O estudo realizado apresentou como limitações o fato de os animais serem expostos ao OMC por apenas 21 dias, tempo suficiente para causar alterações significativas em animais no período pré-lactacional, de acordo com a literatura. O estudo foi realizado no período pós-lactacional, onde os organismos estavam mais desenvolvidos, sendo necessário um tempo maior de exposição para ocasionar mudanças mais significativas nos resultados apresentados. 


\section{REFERÊNCIAS}

1. Garcia EB. Contaminação Ambiental e da cadeia alimentar com filtros solares: um potencial risco à saúde humana. Revista Analytica. 2015; 13(77):45-54.

2. Carnevali O, Maradonna F. Exposure to xenobiotic compounds: looking for new biomarkers. General and Comparative Endocrinology. 2003; 131(3):203-208.

3. Gago-Ferrero P, Cruz, MSD, Barceló D. Occurrence of multiclass UV filters in treated sewage sludge from wasterwater treatment plants. Chemosphere. 2011; 84(8):1158-1165.

4. Dunlap WC, Shick JM, Yamamoto Y. Sunscreens, oxidative stress and antioxidant functions in marine organisms of the Great Barrir Reef. Redox Report. 2013; 4(6):301-306

5. Klammer H, Schlecht C, Wolfgang W, Schmutzler C, Gotthardt I, Kohrle, J, et al. Effects of a 5-day treatment with UV-filter octyl-methoxycinnamate (OMC) on the function of the hypothalamo-pituitary-thyroid function in rats. Toxicology. 2007;238(2):192-199.

6. Sarveiya V, Risk S, Benson HAE. Liquid chromatographic assay for commom sunscreen agents: application to in vivo assessment of skin penetration and systemic absorption in human volunteers. Journal of Chromatography B. 2004; 803(3):231-255.

7. Chapman AK, Farmer, ZJ, Mastrandrea LD, Matlock KA. Neonatal Thyroid Function and Disorders. 2019; 62(2):373-387.

8. Oliveira V, Maldonado RR. Hipotireoidismo e Hipertireoidismo - Uma breve revisão sobre as disfunções tireoidianas. Interciência \& Sociedade. São Paulo. 2014; 3(2):36-45.

9. Bila DM, Dezotti M. Desreguladores endócrinos no meio ambiente: efeitos e consequencias. Química Nova. 2007; 30(3):651-666.

10. Moraes NV, Grando MD, Valerio DAR, Oliveira DP. Exposição ambiental a desreguladores endócrinos: alterações na homeostase dos hormônios esteroidais e tireoideanos. Revista Brasileira de Toxicologia. 2008; 21(1):1-8

11. Schmutzler C, Hamann I, Hofmann PJ, Kovacs G, Stemmler L, Mentrup B, et al. Endocrine active compounds affect tryrotropin and thyroid hormone levels in sérum as well as endpoints of thyroid hormone action in liver, heart and kidney. Toxicology. 2004; 205(1):95-102.
12. Axelstad M, Boberg J, Hougaard KS, Christiansen S, Jacobsen PR, Mandrup KR, et al. Effects of pre- and postnatal exposure to the UV-filter octyl methoxycinnamate (OMC) on the reproductive, auditory and neurological development of rat offspring. Toxicology and Applied Pharmacology. 2011; 250(3):90-278.

13. Watanabe $\mathrm{C}$, Kuwagata M, Shinsuke $\mathrm{Y}$, Azegami J, Kojima $\mathrm{K}$, Ono $\mathrm{H}$, et al. An improved technique for repeated gavage administration to rat neonates. Congenit Anom. Kyoto. 2003;43(3):9-177.

14. Garcia, EB. Efeito da desregulação tireoideana induzida por octil metoxi cinamato sobre parâmetros cognitivos em ratos. [Dissertação de Mestrado]. Rio de Janeiro: Instituto Nacional de Controle de Qualidade em Saúde, Fundação Oswaldo Cruz; 2015. 75p.

15. Nascimento JC, Maleck M, Cruz I, Côrtes PPR. Técnicas histológicas adaptadas para tecidos de ratos. Revista de Saúde. 2020; 11(1):25-28.

16. Freneau GE. Aspectos da morfologia espermática em touros. Revista Brasileira Reprodução Animal. Belo Horizonte. 2015; 39(1):47-60.

17. Axelstad M, Hansen PR, Boberg J, Bonnichsen M, Nellemann C, Lund SP. et al. Developmental neurotoxicity of propylthiouracil (PTU) in rats: relationship between transient hypothyroxinemia during development and longlasting behavioural and functional changes. Toxicology and Applied Pharmacology. 2008; 232(1):1-13.

18. Filipe, JF. Estudo do efeito do Octilmetoxicinamato (OMC) em artérias umbilicais humanas. [Dissertação de Mestrado]. Covilhã: Universidade da Beira Interior; 2016. $26 p$.

\section{CORRESPONDÊNCIA}

Vitor Kunes Lopes

Centro Universitário Barão de Mauá

Rua Martins Reche, 69 - Ribeirão Preto, São Paulo, Brasil CEP: 14056-460

vitor.kunes@hotmail.com 